\title{
Phase stitching and error correction in aperture synthesis for generalized phase-shifting interferometry
}

\author{
Xianfeng Xu, ${ }^{1,2, \star}$ Guangcan Lu, ${ }^{1}$ Guoxia Han, ${ }^{1}$ Fei Gao, ${ }^{1}$ Zhiyong Jiao, ${ }^{1}$ and Dailin Li ${ }^{1}$ \\ ${ }^{1}$ College of Science, China University of Petroleum (East China), Qingdao 266580, China \\ ${ }^{2}$ Bradley Department of Electrical and Computer Engineering, Virginia Tech, Blacksburg, Virginia 24061, USA \\ ${ }^{*}$ Corresponding author: xuxf@upc.edu.cn
}

Received 1 May 2013; revised 10 June 2013; accepted 10 June 2013; posted 11 June 2013 (Doc. ID 189579); published 5 July 2013

\begin{abstract}
An accurate aperture synthesis method in generalized phase-shifting interferometry is suggested to improve the quality of the reconstructed object wavefront by stitching both the phase and the real amplitude of the object wave on the recording plane. Since the phase distribution affects the reconstruction of the original object wavefront, phase stitching is also important in aperture synthesis. Double correlations are used to find the proper relative locations and correct the phase error of subwavefronts on the recording plane. By using phase correction, the phase distributions of subwavefronts are combined perfectly. Corresponding optical experimental results have verified the effectiveness of this method, which can stitch not only the real amplitudes but also the phases of the complex amplitudes of the object wave on the recording plane and improve the quality of the reconstructed object image. (C) 2013 Optical Society of America
\end{abstract}

OCIS codes: (090.0090) Holography; (090.1995) Digital holography; (100.2000) Digital image processing; (120.5050) Phase measurement.

http://dx.doi.org/10.1364/AO.52.004864

\section{Introduction}

In recent years, digital holography [1-5] has attracted many researchers' attention because of its convenience and effectiveness in wavefront measurement. Phase-shifting interferometry is introduced into digital holography to remove the zero-order term and the conjugate image in in-line digital holography $[6,7]$. More than two interferograms are needed for this technique. During the recording process, many environmental factors along with the precision of the phase shifter always cause phase-shift errors, which induce errors on the retrieved object wavefronts [8-11]. Some methods with random phase shifts and many phase-shift retrieval algorithms are useful in decreasing the negative effects of these

$1559-128 \mathrm{X} / 13 / 204864-07 \$ 15.00 / 0$

(C) 2013 Optical Society of America errors [12-20]. Cai et al. [21] developed a generalized phase-shifting interferometry (GPSI) technique for more convenience and high precision in phaseshifting interferometry. Several two-step GPSI methods [22-26] were recently introduced to achieve a higher precision of the extracted phase shift and a higher quality of the reconstructed image.

More recently, researchers [27] have tried to remove such obstacles as the limitations on both the resolution and the size of current recording devices by using two CCDs to enlarge the recording area and gain larger-space frequency spectrums. But some lower-frequency spectrums cannot be recorded, and it is also difficult to set two recording sensors on the same plane without knowing their specific locations. Other researchers also enlarge the recording area by combining of subinterferograms directly. But these methods require more accurate optical devices and impose heavy computation loads on 
computers. We have provided a simple advanced method [28] to enlarge the recording area and improve the resolution of the reconstructed image by using only one CCD.

In all the aperture synthesis approaches mentioned above, the phase stitching of subwavefronts is neither considered nor discussed. In real applications, the phase of the object wave on the recording plane plays an important role in image reconstruction because the phase errors affect not only the phase but also the real amplitude distribution of the wavefront on the original object plane. In fact, the phase of the wavefront is more likely to be influenced by environmental factors. Even a very small device vibration or air disturbance will change the phase value greatly and lead to the failure of phase stitching. In addition, phase combining is more complicated than real amplitude stitching in GPSI.

Here we propose an aperture synthesis method, which can combine both the phase and the real amplitude of the sub-object-wavefronts to provide more convenience and higher precision in wavefront reconstruction. To avoid the influence of environmental factors on intensity distributions of sub-objectwaves, sub-reference-intensity-distributions are employed to find the proper lateral locations of two sub-object-waves through correlation. Phase errors caused by axial movement of devices during lateral movement of the CCD can also be corrected by calculating a phase correlation. Our method can be described as the following operations. First, as the CCD is moved laterally, record several sets of GPSI object, reference, and interfering intensities set by set with small marginal common folded areas [28]. Second, find the proper location through their reference intensity distribution correlations. Third, extract their phase shifts and reconstruct their subwavefronts on the recording plane by a two-step GPSI algorithm. Fourth, correct the sub-phase-distributions and combine sub-objectwavefronts on the recording plane. Fifth, reconstruct the objective wavefront by a convolution method. For convenience and simplicity, two sets of subframes are used as an example to illustrate the suggested method, where the two sets of intensity distributions are denoted $I_{o 1}, I_{r 1}, I_{11}, I_{21}$, and $I_{o 2}, I_{r 2}, I_{12}, I_{12}$. In the following sections, we present sequentially the subframe matching, subwavefront reconstruction, phase correction, and original object wave reconstruction, optical experimental verification, and conclusions.

\section{Subframe Matching}

In our previous work [28], to avoid the influence of device vibration and oscillation on the stability of interferograms and secure the success of aperture synthesis, correlation of sub-object-intensities was employed to find the relative location, and then the subwavefronts were retrieved and stitched, without using the combination of interferograms. In that work, the object intensity distributions were used as reference to find the relative location of subwavefronts, because the object wave intensities change very little when the recording distance changes slightly. Since the intensity distributions of the reference beam are more stable during the recording process even when the object moves or oscillates slightly, it is a better choice to use the reference intensity as a reference to search for the actual positions of subwavefronts. In fact, there are many marks on the reference beam due to the defects of the combiner, although the reference intensity should be constant in theory.

By using the analysis above, we can stitch several sub-object-waves by referring to the reference intensity. For convenience and simplicity, the method is illustrated by using two-step GPSI methods.

In the folded area of two subreferences $I_{r 1}$ and $I_{r 2}$, a model with $m \times n$ pixels is selected in the bottom center of $I_{r 1}$ and denoted $i_{r 1}\left(x_{1}, y_{1}\right)$. Supposing that an area $i_{r 2}\left(x_{2}, y_{2}\right)$ with the same size of $i_{r 1}$ is chosen in the folded part of $I_{r 2}$, their correlation value can be written as

$$
c_{i}(x, y)=i_{r 1}\left(x_{1}, y_{1}\right) \oplus i_{r 2}\left(x_{2}, y_{2}\right),
$$

where the symbol $\oplus$ represents the correlation of two subframes $i_{r 1}$ and $i_{r 2}$ and $\left(x_{1}, y_{1}\right),\left(x_{2}, y_{2}\right)$, and $(x, y)$ are the coordinates of $i_{r 1}, i_{r 2}$, and the position of the first pixel [with coordinates $(1,1)$ ] of $i_{r 1}$ in $I_{r 2}$. The correlation value $c_{i}$ varies with the relative location of $i_{r 1}$ in $I_{r 2}$, and naturally the maximum value of $c_{i}$ corresponds to the actual relative position of $i_{r 1}$ in $I_{r 2}$, which can be denoted by the index coordinate $\left(x_{\max }, y_{\max }\right)$. We can calculate the value of $c_{i}(x, y)$ by iteration and then find the maximum value $c_{i \max }$ with its corresponding coordinate $\left(x_{\max }, y_{\max }\right)$, which is used as the index for subwavefront combination.

\section{Subwavefront Reconstruction on Recording Plane}

If we record the two sets of GPSI [24] subframes including objective, reference, and two interfere intensities $I_{o 1}, I_{r 1}, I_{11}, I_{21}$ and $I_{o 2}, I_{r 2}, I_{12}, I_{22}$, their phase shifts can be extracted by

$$
\begin{gathered}
\alpha_{q}=2 \tan ^{-1}\left[\frac{\left\langle\left(I_{1 q}-I_{2 q}\right) /\left(4 \sqrt{I_{o q} I_{r q}}\right) \|\right.}{\left\langle\left(I_{1 q}+I_{2 q}-2 I_{o q}-2 I_{r q}\right) /\left(4 \sqrt{I_{o q} I_{r q}}\right) \mid\right\rangle}\right], \\
q=1,2 .
\end{gathered}
$$

In Eq. (2), consequently, the subscript $q$ represents the set number of subframes. The object wave on the recording plane can be reconstructed as

$$
\begin{aligned}
U_{q}= & \frac{I_{1 q}-I_{o q}-I_{r q}}{2 \sqrt{I_{r q}}} \\
& +i \frac{I_{2 q}-I_{1 q} \cos \alpha_{q}-\left(1-\cos \alpha_{q}\right)\left(I_{o q}+I_{r q}\right)}{2 \sqrt{I_{r q}} \sin \alpha_{q}} .
\end{aligned}
$$


During the GPSI recording process, the intensities $I_{o 1}, I_{r 1}, I_{11}, I_{21}$ and $I_{o 2}, I_{r 2}, I_{12}, I_{22}$ are not captured at the same time. Intensity distributions $I_{o 1}, I_{r 1}$ and $I_{o 2}, I_{r 2}$ change very little, but $I_{11}, I_{21}$ and $I_{12}, I_{22}$ change greatly because of the phase shifts of the reference. So it is difficult for us to combine the interferograms $I_{11}$ and $I_{12}$ or $I_{21}$ and $I_{22}$. The reported method [28] that retrieves the subwavefronts first and then combines them on the recording plane is an effective approach to complete the aperture synthesis in GPSI.

\section{Phase Stitching and Original Wavefront Reconstruction}

Although the sub-object-intensities change very little at different time during recording process according to our analysis, the phases of the sub-object-wavefronts on recording plane will change greatly with the relative distance variation between the object and CCD chip. So the phase combination should be considered in the process of aperture synthesis.

Assuming that the CCD chip moves a small axial distance $d$ between the first set and the second set of GPSI recordings, the phase of the second subwavefront will change

$$
\Delta \varphi=2 \pi d / \lambda .
$$

In Eq. (4), $\lambda$ is the wavelength of the light source. If the phases of two subwavefronts in the folded area are denoted $\varphi_{o 1}$ and $\varphi_{o 2}$ separately, then

$$
\varphi_{o 2}=\varphi_{o 1}+2 \pi d / \lambda .
$$

It is easy to see that the object phase $\varphi_{o 2}$ changes significantly if the value of $d$ reaches the magnitude of a micro. However the small axial displace $d$ is caused by the random movement of the CCD, and it is difficult to measure $d$ precisely. Naturally, without knowing $d$, it is impossible to know the object phase difference $\varphi_{o 2}-\varphi_{o 1}$. How can the error of the phase distribution of $\varphi_{o 2}$ be corrected?

Although the specific value of $d$ is not known in practice, it is constant for all the pixels on the CCD plane because the movement is mainly in the axial direction and the nonaxial movement is trivial. In the folded common area, the two subwavefronts retrieved should have the same object phase distributions if there is no axial displacement between the object and the CCD. In this situation, the two sub-phase-distributions should have the biggest correlation value. But there is mostly axial movement of the CCD, and the phase error $\Delta \varphi$ appears in the second subwave. Here the phase term is periodic, so the value of $\Delta \varphi$ can be limited in the period of $(0,2 \pi)$ for the purpose of phase correction. If the phase difference is written as $\Delta \varphi_{o}$, the relationship of the two sub-object-phases in common area can be expressed as

$$
\varphi_{o 2}=\varphi_{o 1}+\Delta \varphi_{o}
$$

The value of $\Delta \varphi_{o}$ can be found by calculating the correlation

$$
c_{p}(x, y)=\varphi_{o 1}\left(x_{1}, y_{1}\right) \oplus\left[\varphi_{o 2}\left(x_{2}, y_{2}\right)-\Delta \varphi_{o}\right] .
$$

To find the true error for $\Delta \varphi_{o}$, the values of different $\Delta \varphi_{o}$ are selected in a $(0,2 \pi)$ period with equal intervals to calculate $c_{p}$ according to formula (7). Obviously, the value of $\Delta \varphi_{o}$ corresponding to maximum correlation value $c_{p \max }$ is the right $\Delta \varphi_{o}$. By using Eq. ( $\left.\underline{6}\right)$ and the right $\Delta \varphi_{o}$, the phase error of the second subwavefront can be corrected. Then the two subphases can be stitched together with the same coordinate index from their two sub-reference-waves' correlation.

After the stitching of phase and real amplitude of sub-object-wavefront with coordinate index and corrected phase, the object wavefront can be synthesized altogether as $U$. By convolution reconstruction, the original object wavefront can be calculated as

$$
O=\operatorname{IFFT}[\operatorname{FFT}(h) \operatorname{FFT}(U)],
$$

where IFFT and FFT are the inverse Fourier and Fourier transforms, respectively, and

$$
h=\exp (i k z) \exp \left[i k\left(x^{2}+y^{2}\right) /(2 z)\right] /(i \lambda z),
$$

where $z$ is the recording distance and $k$ is the wave vector.

The whole process of our algorithm is illustrated by a flow chart in Fig. 1 . After the two sets of frames are recorded, the phase shifts are extracted and the subwavefronts $U_{1}$ and $U_{2}$ on the recording plane are reconstructed by Eqs. (2) and (3), respectively. By using the two sub-reference-intensities and Eq. (1), the proper coordinates $\left(x_{\max }, y_{\max }\right)$ are determined. Then the phase of the second subwavefront is corrected by Eq. (6) and the two subwavefronts are stitched together on the recording plane. Finally, the synthesized wavefront on the object plane is obtained by Eq. (ㅇ).

Although we discussed sub-object-phase combination in aperture synthesis with the example of two

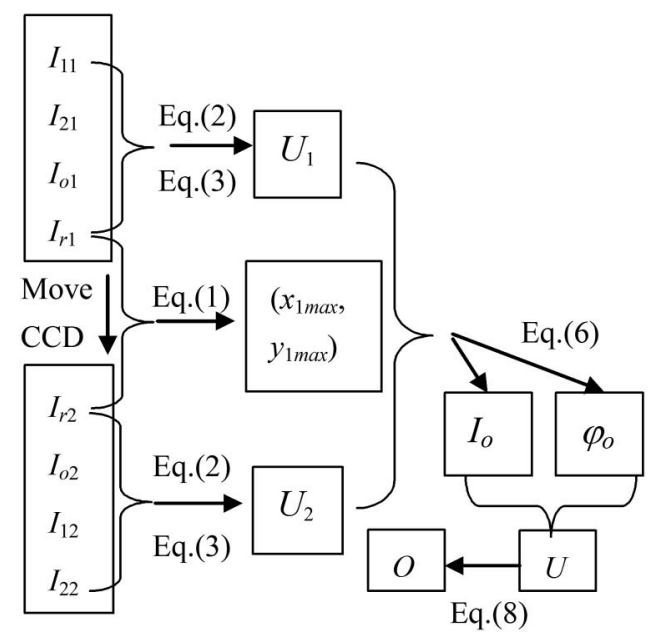

Fig. 1. Flow chart for complex amplitude synthesis. 
sets of subframes in GPSI, obviously this algorithm can be extended to the condition of any subframe number greater than two by combining the two combined ones further. Once all the sub-objectwavefronts are combined, the original object wave can be retrieved.

\section{Optical Experiments}

Some optical experiments have been carried out to investigate the performance of this phase stitching method in practice. The experimental setup is shown in Fig. 2, which is similar to that given in [25], but with a moveable CCD device, and the resolution target is replaced by a slightly divergent spherical wave on original object plane. The wavelength of the $\mathrm{He}-$ $\mathrm{Ne}$ laser used is $632.8 \mathrm{~nm}$. The light beam first passes reflector $\mathrm{M}_{1}$ and then is divided by the beam splitter $\mathrm{BS}_{1}$ into two light beams that are filtered and collimated as the reference and the objective light beam, respectively. The recording area of the CCD with pixel size $6.45 \mu \mathrm{m} \times 6.45 \mu \mathrm{m}$ is restrained to the central part of $896 \times 1252$ pixels. The recording distance is set as $z=9.1 \mathrm{~cm}$. Two sets of GPSI frames with a common folded area are recorded by changing the position of the CCD on the recording plane. We show only the two reference intensities and two interferograms of the upper set and the lower set frames in Figs. 3(a), 3(c) and Figs. 3(b), 3(d), respectively. To find the relative matching position for two reference intensities, a small model $i_{r 1}$ with $128 \times 1024$ pixels is chosen from the central bottom of the folded area in $I_{r 1}$ [see Fig. 4(a)]. A small area $i_{r 2}$ with the same size as $i_{r 1}$ selected in $I_{r 2}$ is used to calculate the correlation value by Eq. (1). By changing the relative coordinates of the first pixel of $i_{r 1}$ in $I_{r 2}$, Coordinate $\left(x_{\max }, y_{\max }\right)$ is searched by iteration, and the results is $x_{\max }=125$ pixels, $y_{\max }=365$ pixels, $c_{i \max }=0.6547$. To present the distributions of $c_{i}$ with $(x, y)$ and $(x, 365)$, the calculation results are given in Figs. $\underline{5}$ and $6(\mathrm{a})$, respectively. In Figs. 5 and $6(\mathrm{a})$, the maximum correlation value is about $0.654 \overline{7 \mathrm{at}}$ the matching coordinate, but other correlation values

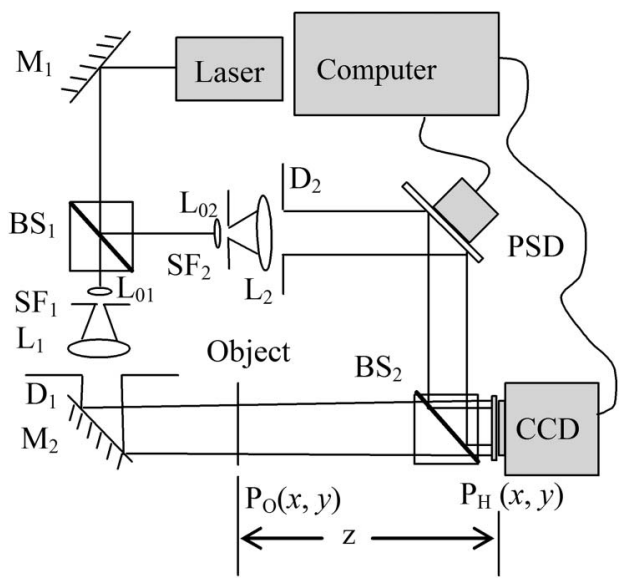

Fig. 2. Experiment setup for aperture synthesis. SF, space filter; $\mathrm{L}$, lens; $\mathrm{D}$, diaphragm; $P_{O}$, object plane; $P_{H}$, recording plane; $\mathrm{PSD}$, phase-shifting device. decrease quickly to below 0.2 . If we choose the model with a higher pixel number, the value of $c_{i \max }$ becomes larger, but more calculating time is needed. In fact, the lower the pixel number in the model, the faster we calculate the correlation value. When we decrease the model pixel number to $128 \times 8$, the peak on the curve of $c_{i}$ is still available and little computation time is needed. So this correlation method is effective in finding the real relative positions of subframes.

The two phase shift values $\alpha_{1}$ and $\alpha_{2}$ are extracted by Eq. (2) with two sets of frames, and then two sub-object-complex-waves $U_{1}$ and $U_{2}$ are further retrieved with Eq. (3). The phases and intensities of the two object wavefronts reconstructed by Eq. (8) on the original object plane through the convolution method are shown in Figs. 7(a), 7(c) and Figs. 7(b), 7(d), respectively. In these phase maps, the lower part of Fig. $\underline{7 \text { (a) }}$ and the upper part of Fig. $\underline{7(\mathrm{c})}$

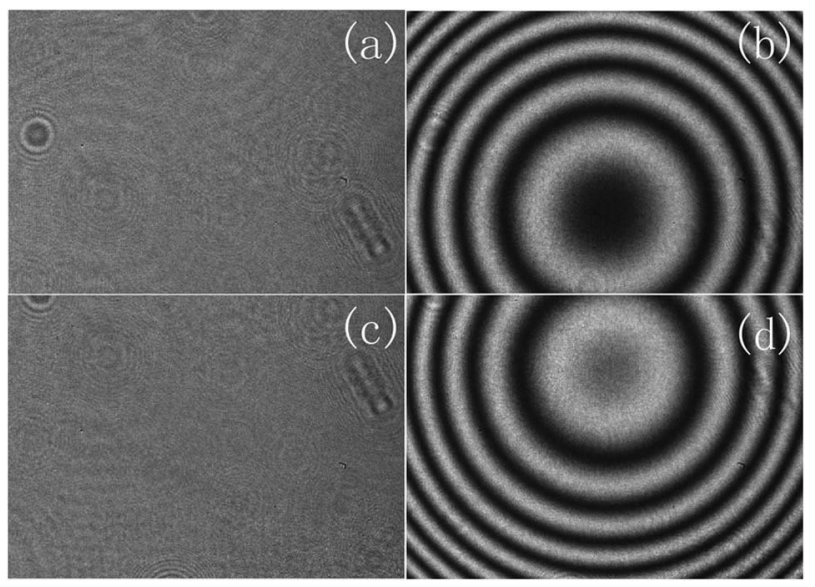

Fig. 3. Reference intensities and interferograms (a), (b) from the upper and (c), (d) from the lower set of GPSI frames.

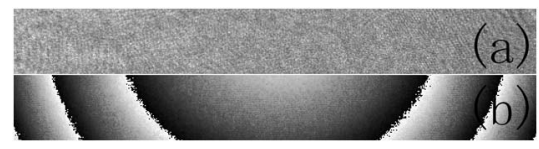

Fig. 4. Correlation models: (a) intensity matching model and (b) phase correction model.

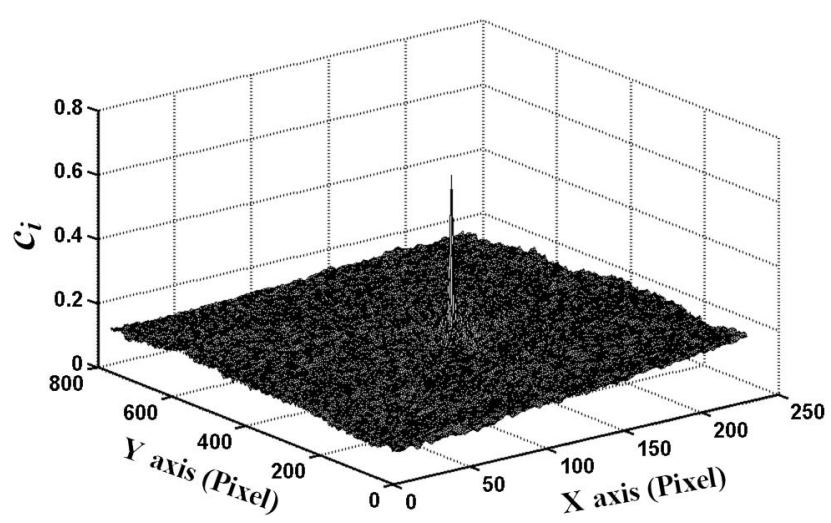

Fig. 5. Correlation value distributions on the recording plane. 

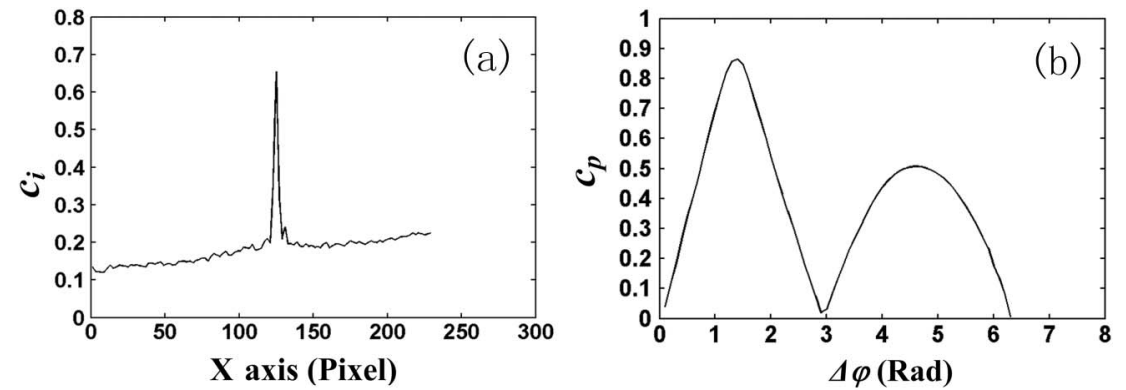

Fig. 6. Correlation value distributions (a) in one direction and (b) for phase correction.

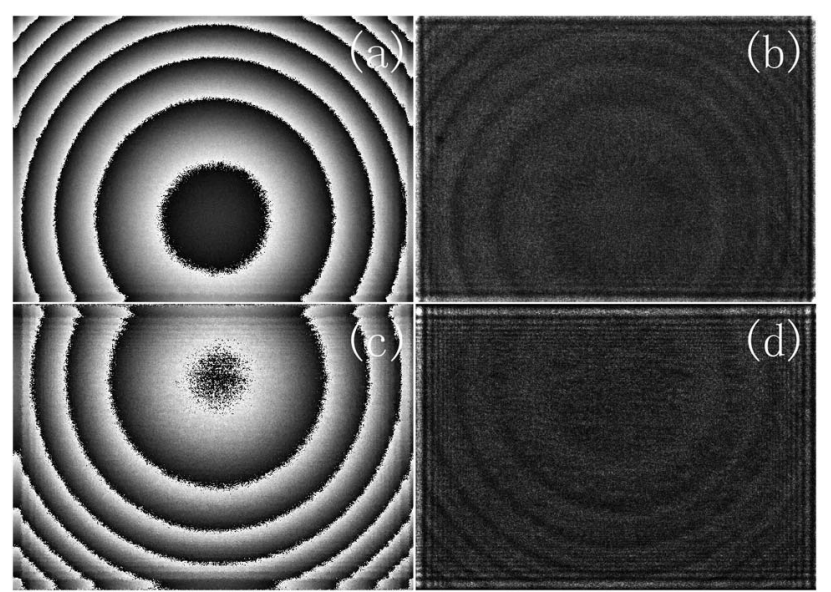

Fig. 7. Phases and intensities of object wavefront retrieved on original plane (a), (b) from the upper set of frames and (c), (d) from the lower set of frames.

have different phase distributions. If we combine the two object wavefronts $U_{1}$ and $U_{2}$ directly by reference to only the coordinate $\left(x_{1 \text { max }}, y_{1 \text { max }}\right)$, there will be phase errors in the new synthesized wavefront. We stitched $U_{1}$ and $U_{2}$ together into a new $U$ with $1300 \times 1262$ pixels and padded the vacant area with zeros. Figures $8(a)$ and $8(b)$ show us the phase and intensity of the upper part of $1262 \times$ 1262 pixels of the objective wavefront reconstructed on the recording plane. The intensities are stitched together well in Fig. 8(b), but the phase strips in Fig. 8(a) are not normal circles because of the phase error of $U_{2}$. The convolution method is used to reconstruct the wavefront $O$ on the original object plane. Figures 9(a) and 9(b) show us the phase and intensity distributions of $O$ without error correction. The phase circles in Fig. 9(a) are also deformed, and there is a dark rectangular area around the horizontal center line of Fig. 9(b). Obviously, not only the phase map, but also the intensity distribution is affected by the object phase error on the recoding plane, which was analyzed in $[29,30]$. To correct the phase error of $U_{2}$, a model [see Fig. 4(b)] with $128 \times 1024$ pixels is selected from the phase $\varphi_{o 1}$ of $U_{1}$ in the folded area, and the corresponding part is defined from $\varphi_{o 2}$ of $U_{2}$ to calculate their correlation value. By using Eq. (7) and changing the value of $\Delta \varphi_{o}$ in iterations, we calculated 100 different values of $\Delta \varphi_{o}$ distributed equally in $(0,2 \pi)$. Figure $6(\mathrm{~b})$ gives us

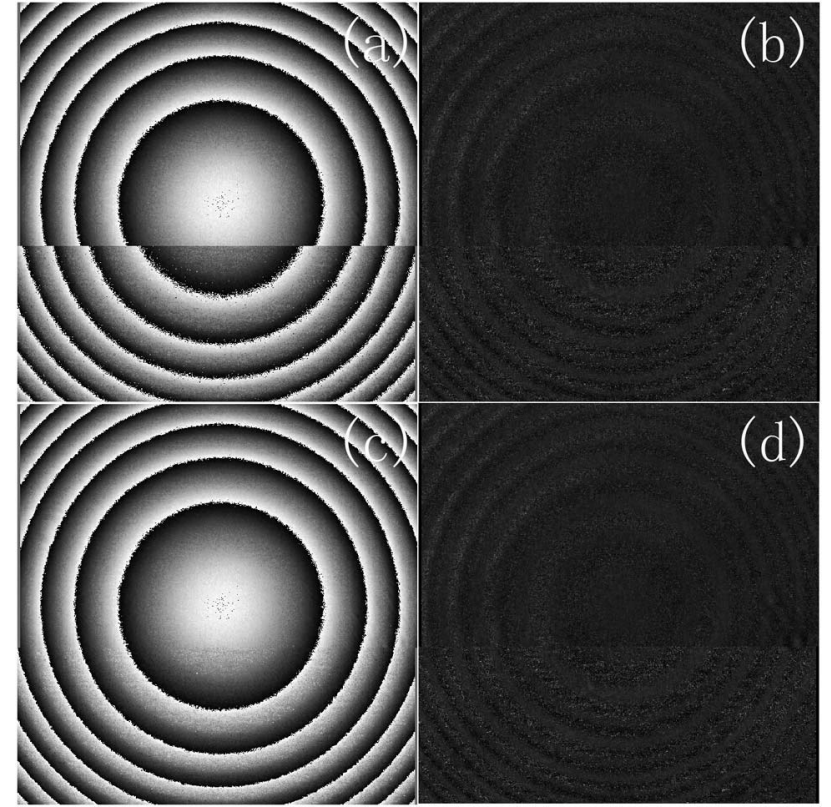

Fig. 8. Phases and intensities of the object wave combined on recording plane (a), (b) without phase correction and (c), (d) with phase correction.

the curve of $c_{p}$, and in fact the biggest correlation value is $c_{p}=0.8642$, which corresponds to $\Delta \varphi_{o}=1.34 \mathrm{rad}$. The maximum value of $c_{p}$ approaches 1 (the maximum value in theory). But for other values of $\Delta \varphi_{o}, c_{p}$ decreases sharply to zero and then reaches a lower peak of 0.5 , which is different from the distribution trend of reference intensity correlation value $c_{i}$. We use $\Delta \varphi_{o}=1.34 \mathrm{rad}$ and Eq. ( $\underline{6})$ to correct the phase of $U_{2}$, and Fig. 8(c) shows the synthesized phase, which is much better than that in Fig. 8(a). The corresponding phase and intensity of the wavefront calculated by the convolution method are given in Figs. 9(c) and 9(d), respectively. It is easy to see that the phase map is regular concentric circles and the intensity distribution is almost a unit value, which reflects a slightly divergent spherical wavefront. Because of the negative effect of some error sources other than phase error, there are some residue fringes in Fig. 9(d). But the dark stripe in Fig. 9(b) has been removed after phase correction. So, by such phase correction, we improved the quality of the aperture synthesis in GPSI. In our previous 


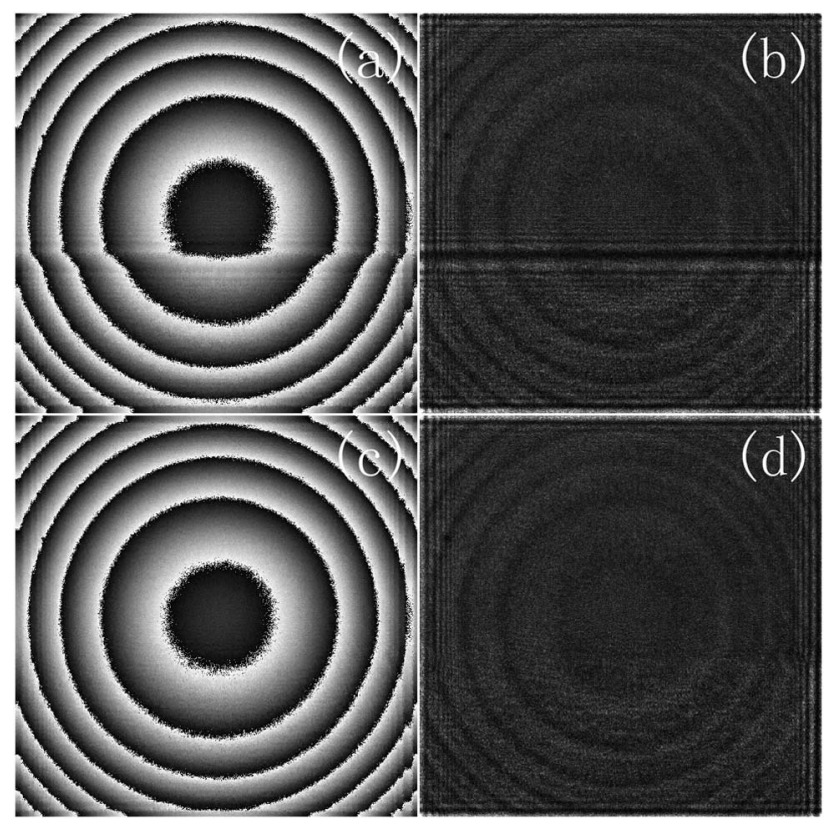

Fig. 9. Phases and intensities of object wave on original plane (a), (b) without phase correction and (c), (d) with phase correction.

work [28], only intensity was considered and the phase error was omitted. For the intensity object, the former method reported [28] works well. But for a pure phase object and an object with both amplitude and phase distributions, the method provided here is needed.

Our many other experiments using different phase objects have shown the same trends discussed above.

\section{Conclusions}

In summary, an effective method to achieve aperture synthesis by considering not only intensity but also phase distributions to combine the sub-object-wavefronts on the recording plane in two-step GPSI is provided. The specific procedures of this method can be shortened into the following steps. Step 1, several sets of interferograms with marginal folded areas are recorded on the different areas of the recording plane. Step 2, a model is selected from one reference intensity in the folded area, and the stitching location is found by calculating the correlation values between the model and the corresponding area in the other sub-reference-intensity. Step 3, phase shifts are extracted and sub-object-waves are reconstructed separately. Step 4, by using the index coordinate, the phase errors are corrected by the correlation method, and the sub-object-waves are combined. Step 5, the object wavefront is reconstructed by the convolution method on the original object plane. The optical experiments with a slightly divergent spherical wavefront show that the present method is effective in aperture synthesis for both intensity and phase objects. Although only two sets of sub-GPSI-frames are combined as an example, more sets of sub-interference-frames can also be synthesized by this method. By stitching both the intensity and the phase of the sub-object-waves, this technique can improve the quality of the retrieved holographic image. We believe that this technique will find useful applications in future work, especially when large-size phase objects need to be recorded. The excellent performance of this method promises very prosperous applications in further practice.

This work is supported by the National Natural Science Foundation of China under grant 60977006, Studying Abroad Scholarships of China, the Fundamental Research Funds for the Central Universities of China under grants 12CX04086A and 24720146005A, and the Natural Science Foundation of Shandong Province, China under grants ZR2009GM016 and ZR2012DL12.

\section{References}

1. J. W. Goodman and R. W. Lawrence, "Digital image formation from electronically detected holograms," Appl. Phys. Lett 11, 77-79 (1967).

2. T. C. Poon, T. Akin, G. Indebetouw, and T. Kim, "Horizontalparallax-only electronic holography," Opt. Express 13, 2427-2432 (2005).

3. P. Tsang, W. K. Cheung, T. C. Poon, and C. Zhou, "Holographic video at 40 frames per second for 4-million object points," Opt. Express 19, 15205-15211 (2011).

4. M. K. Kim, "Adaptive optics by incoherent digital holography," Opt. Lett. 37, 2694-2696 (2012).

5. T. Kim and T. C. Poon, "Autofocusing in optical scanning holography," Appl. Opt. 48, H153-H159 (2009).

6. I. Yamaguchi and T. Zhang, "Phase-shifting digital holography," Opt. Lett. 22, 1268-1270 (1997).

7. Y. C. Lin, C. J. Cheng, and T. C. Poon, "Optical sectioning with a low-coherence phase-shifting digital holographic microscope," Appl. Opt. 50, B25-B30 (2011).

8. Y. Surrel, "Additive noise effect in digital phase detection," Appl. Opt. 36, 271-276 (1997)

9. J. Schmit and K. Creath, "Window function influence on phase error in phase-shifting algorithms," Appl. Opt. 35, 5642-5649 (1996).

10. B. Gutman and H. Weber, "Phase-shifter calibration and error detection in phase-shifting applications: a new method," Appl. Opt. 37, 7624-7631 (1998).

11. J. Novák, P. Novák, and A. Mikš, "Multi-step phase-shifting algorithms insensitive to linear phase shift errors," Opt. Commun. 281, 5302-5309 (2008).

12. J. E. Greivenkamp, "Generalized data reduction for heterodyne interferometry," Opt. Eng. 23, 234350 (1984).

13. G. Lai and T. Yatagai, "Generalized phase-shifting interferometry, J. Opt. Soc. Am. A 8, 822-827 (1991).

14. X. Chen, M. Gramaglia, and J. A. Yeazell, "Phase-shifting interferometry with uncalibrated phase shifts," Appl. Opt. 39, 585-591 (2000).

15. A. Patil, B. Raphael, and P. Rastogi, "Generalized phaseshifting interferometry by use of a direct stochastic algorithm for global search," Opt. Lett. 29, 1381-1383 (2004).

16. J. C. Xu, Q. Xu, L. Q. Chai, Y. Li, and H. Wang, "Direct phase extraction from interferograms with random phase shifts," Opt. Express 18, 20620-20627 (2010).

17. H. W. Guo, "Blind self-calibrating algorithm for phase-shifting interferometry by use of cross-bispectrum," Opt. Express 19, 7807-7815 (2011).

18. B. C. Chen and C. Basaran, "Statistical phase-shifting step estimation algorithm based on the continuous wavelet transform for high-resolution interferometry metrology," Appl. Opt. 50, 586-593 (2011).

19. J. C. Xu, W. M. Jin, L. Q. Chai, and Q. Xu, "Phase extraction from randomly phase-shifted interferograms by combining 
principal component analysis and least squares method," Opt. Express 19, 20483-20492 (2011).

20. R. Juarez-Salazar, C. Robledo-Sánchez, C. Meneses-Fabian, F. Guerrero-Sánchez, and L. M. Arévalo Aguilar, "Generalized phase-shifting interferometry by parameter estimation with the least squares method," Opt. Lasers Eng. 51, 626-632 (2013).

21. L. Z. Cai, Q. Liu, and X. L. Yang, "Generalized phase-shifting interferometry with arbitrary unknown phase steps for diffraction objects," Opt. Lett. 29, 183-185 (2004).

22. X. F. Xu, L. Z. Cai, Y. R. Wang, X. L. Yang, X. F. Meng, G. Y. Dong, X. X. Shen, and H. Zhang, "Generalized phase-shifting interferometry with arbitrary unknown phase shifts: direct wavefront reconstruction by blind phase shift extraction and its experimental verification," Appl. Phys. Lett. 90, 121124 (2007).

23. X. F. Xu, L. Z. Cai, Y. R. Wang, X. F. Meng, W. J. Sun, H. Zhang, X. C. Cheng, G. Y. Dong, and X. X. Shen, "Simple direct extraction of unknown phase shift and wavefront reconstruction in generalized phase-shifting interferometry: algorithm and experiments," Opt. Lett. 33, 776-778 (2008).

24. X. F. Xu, L. Z. Cai, Y. R. Wang, and D. L. Li, "Accurate phase shift extraction for generalized phase-shifting interferometry," Chin. Phys. Lett. 27, 024215 (2010).
25. X. F. Xu, L. Z. Cai, Y. R. Wang, and R. S. Yan, "Direct phase shift extraction and wavefront reconstruction in two-step generalized phase-shifting interferometry," J. Opt. 12, 015301 (2010).

26. X. F. Xu, G. C. Lu, Y. J. Tian, G. X. Han, H. G. Yuan, F. Gao, X. X. Miao, and Z. Y. Jiao, "High-precision phase-shifting interferometry with spherical wavefront reference," Appl. Opt. 52, A188-A194 (2013).

27. T. Kreis and K. Schlüter, "Resolution enhancement by aperture synthesis in digital holography," Opt. Eng. 46, 055803 (2007).

28. X. F. Xu, L. Z. Cai, G. C. Lu, G. X. Han, Y. J. Tian, Q. Zhang, and Z. Y. Jiao, "Advanced Aperture synthesis by wave-front combination in generalized phase-shifting interferometry," Chin. Phys. Lett. 29, 084202 (2012).

29. L. Z. Cai, Q. Liu, and X. L. Yang, "Simultaneous digital correction of amplitude and phase errors of retrieved wavefront in phase-shifting interferometry with arbitrary phase shift errors," Opt. Commun. 233, 21-26 (2004).

30. X. F. Xu, L. Z. Cai, Y. R. Wang, X. F. Meng, X. C. Cheng, H. Zhang, G. Y. Dong, and X. X. Shen, "Correction of wavefront reconstruction errors caused by light source intensity instability in phase-shifting interferometry," J. Opt. A 10, 085008 (2008). 